\title{
Mine Safety Monitoring System Based on Wireless Sensor Network
}

\author{
Junwen Zhang*, Shuai Zhong, Zhuqing Liang \\ Lanzhou Resource and Environment Voc-Tech College, Electrical and Mechanical Department, Lanzhou, \\ 730021, China. \\ *corresponding author
}

Keywords: Wireless Sensors, Coal Mine, Safety Monitoring, Systems.

\begin{abstract}
Coal work requires workers to carry out construction under the mine, because of the complexity of the underground environment, if the safety protection work is not done, it is easy to cause coal mine safety accidents. In order to ensure the smooth progress of coal operation and provide safety guarantee for workers, it is necessary to fully understand the specific conditions in the environment under the mine, including humidity, temperature, gas concentration and so on. In recent years, with the continuous progress of science and technology, the wireless sensor network security detection system has been widely used in coal mine safety detection, which plays a vital role in the mine structure is becoming more and more complex, and the tunnel tunnel is becoming more and more narrow.
\end{abstract}

\section{Introduction}

The coal industry is dangerous and complex, so it is necessary for the workers to go deep into the mine to carry out the construction, and the dust, environment, top pressure and fire in the underground environment will threaten the life safety of the workers. As a leading coal-producing country in the world, coal safety incidents occur frequently in recent years, as shown in figure 1 , which is the first aid scene of fire in coal mining industry in hunan. caused widespread concern of the community. In response to this problem, the coal industry needs to take measures to strengthen prevention. In order to fundamentally reduce the coal mine safety incidents, we need to fully understand the specific situation of the underground environment. Therefore, it has become the primary task of coal industry to supervise the coal mine safety system and improve the work safety under the mine[1]. In recent years, with the continuous progress of science and technology, wireless sensor network has been gradually applied to coal mine safety detection, and the detection effect is accurate and reliable, which not only effectively reduces coal mine safety accidents, but also improves the efficiency and level of coal mine operation in an all-round way, thus promoting the stable development of coal industry.

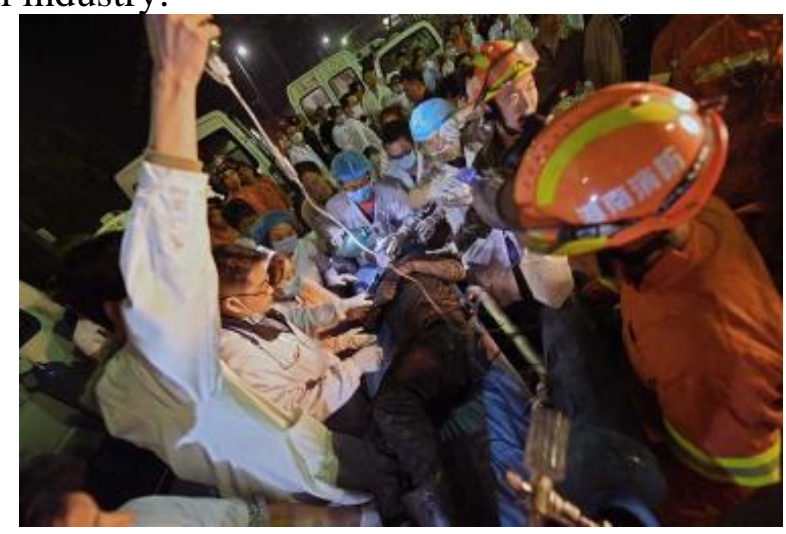

Figure 1 Hunan coal mine safety accident first aid scene

\section{Structure of Wireless Sensor Network}

In recent years, with the continuous development of science and technology, the scale of 
development of sensor technology, information processing technology and other fields has gradually expanded, which has laid a good foundation for wireless sensor network. The so-called wireless sensor network is a network system organized and composed by various ways. Sensors can detect the surrounding environment through infrared, radar and so on.[2] to obtain the humidity, temperature, dust and other data in the environment through the signal wave, and have the accuracy and reliability.

The structure of wireless sensor network involves a wide range of aspects, including sensor node, convergent node and management node. In general, sensor node deployment will be close to the monitoring area, and then the sensor network system will be formed by the way of self-organization. In addition, the data detected by sensor node will usually be transmitted one by one along other sensor nodes. During the transmission of sensor node, multiple nodes will process the detected data, and the detected data will arrive at the convergent node after multiple hops. transport it to the management node. In favour Users Achieving The management nodes configure and manage the sensor network The goal of enabling Release Scientific and accurate Monitoring data.

\section{Design of Coal Mine Safety Monitoring System Based on Wireless Sensor Network}

\subsection{Design Options}

In the design process of coal mine safety monitoring system with wireless sensor network, the system can be divided into two parts: ground monitoring system and underground information collection system. Among them, the ground monitoring system needs to make statistics, processing and analysis of the data transmitted by the wireless sensor network. The underground information acquisition system uses sensors to detect the underground environment and send the data to the gathering point. Then there are convergence points to collate the data, and send the data to the ground control system[3]. so as to lay a good foundation for the smooth construction of the workers.

\subsection{Design of Sensor Network Node}

In terms of hardware structure design, it can be divided into the following four modules. They are: processor module, wireless communication module, energy supply module and sensor module. Among them, the main task of the processor module is to collect data and analyze data, thus laying a good foundation for communication, computing and other tasks, which plays an important role in the design of sensor network nodes. Wireless communication module, as the name implies, the main task is to be responsible for data communication work, while coordinating the signal transmission mode and transmission speed in communication. energy supply module, the main task of this module is power supply work, which can directly affect the normal operation of the sensor network. And the sensor module, the main task is to sense the surrounding information, generally can be divided into sensor probe and transmission system two slow down. The luminosity and temperature in the environment can be monitored by probe [4]. the monitoring data is sent to the processor module by the transmitter processing.

\subsection{Software Design Scheme}

In the design of sensor network node network, it can be composed of the following three parts: communication protocol system, routing protocol system and embedded operating system. It can also be summed up in two parts, one being system-oriented. the other part is customer-oriented layer. specific as shown in Figure 2.

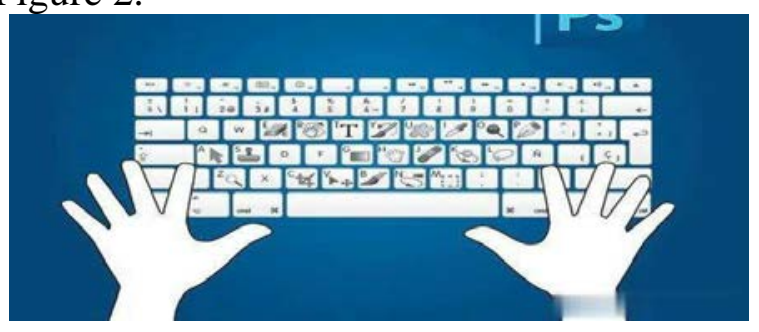

Figure 2 Basic structure of software design 


\subsection{Operating System Design}

Mine Safety Monitoring System for Wireless Sensor Network The application program can be implemented directly in the hardware, but there are some problems, which can be reflected in the following two aspects. First, it is necessary to program the hardware in advance, which is challenging, and the programming effect is different from the traditional operating system, and the function service is less.[5]. Second, the closed shop with low software utilization, because the software results cannot be inherited in the operation process, so it needs to be operated by the way of each restart. Based on this, it is necessary to establish an independent operating system on the sensor node.

There are two ways to establish an independent operating system in the sensor node, one is an embedded operating system, which needs to be applied in combination with the sensor node and tailor the core of the system, thus simplifying the operating system and promoting the normal operation of the system. The other is the WSN operating system, which is based on the development of network technology, has rich functionality, and can give full play to the characteristics of hardware, and has the effect of energy saving. 4。 Node Selection of Coal Mine Safety Monitoring System Based on Wireless Sensor Network

In the process of selecting sensor nodes, it is necessary to fully consider the use environment, because of the particularity of the environment under the coal industry, it needs to be compared and tested many times in the selection of sensor network nodes, which can be analyzed from the following points: the first type selection is CC100 module, the working frequency of this module is adjustable, usually about $442 \mathrm{MHZ}$, and the frequency can reach $77.6 \mathrm{~kb}$ per second.

The second type selection is CC1110 module, this module, with unique advantages, including low cost, low efficiency and other reverse, with a processor and wireless communication transceiver composition.

In order to ensure that the selection of nodes is scientific, it is necessary to compare them repeatedly through performance tests. The main contents of the tests include packet loss rate and packet acceptance, so as to ensure that the selection of nodes in wireless sensor networks is scientific.

\section{Routing Protocol Design of Coal Mine Safety Monitoring System Based on Wireless Sensor Network}

Underground environmental monitoring needs to ensure the accuracy and reliability of monitoring data. In order to improve the service quality and efficiency of communication, we need to improve the design level of routing protocol. In the specific design process, we should fully follow the following aspects:

It is necessary to ensure that the routing protocol has the energy efficiency and extends the network service life as far as possible.

It is necessary to ensure the reliability of the routing protocol so as to guarantee the accuracy of data transmission.

It is necessary to ensure that the routing protocol has real-time, so as to realize the final mode number of real-time monitoring and scientific positioning.

In the actual design process, we should strictly follow the routing protocol to carry out the analysis, first of all, we need to choose the routing protocol with energy. Thus, the network load is distributed equally to the sensor nodes, and the energy consumption is reduced effectively[6]increase the network survival time. Secondly, it is necessary to combine the remaining energy of the node to establish a scientific energy information table. finally, select the node with the most energy and use it as the jump point in the next section. If we know the location of the neighbor node energy, we can scientifically select the node, establish the network master path, complete this step, and use other nodes to expand the path. As shown in figure 3: 


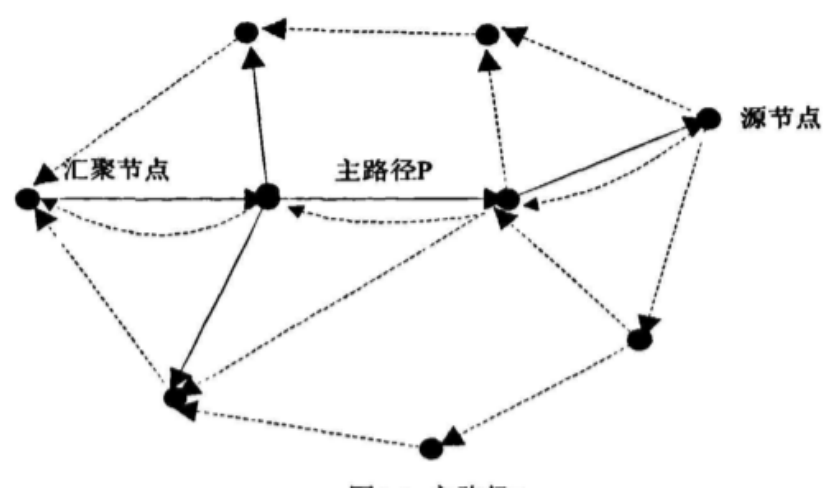

Figure 3 Main road map

\section{Clustering}

The so-called clustering establishment refers to sending each node to the cluster head node through the protocol after clustering itself. To achieve this, you need to create a transfer schedule. First of all, after the cluster data confirmation sends the message, the head node needs to combine the total number of nodes to automatically generate the schedule. At the same time, all the nodes in the timetable will be able to ensure that each node can achieve the goal of data transmission while allocating its own time slot. in addition, if entering the dormancy time at other times, it can effectively reduce the energy consumption generated in the transmission process. only by fully satisfying these conditions, can the protocol be guaranteed to have stability.

\section{Conclusion}

To sum up, China is a big country of coal, coal industry as a special industry, with complexity and danger, workers in the deep under the mine, affected by dust, fire, flood and so on will directly threaten the life of workers. According to statistics, in the process of coal operation in China, the probability of natural fire accident is about $53 \%$, and the explosion accident is about $88 \%$. In order to effectively reduce the probability of coal accidents, it is necessary to establish a coal mine safety monitoring system and make full use of science and technology. In recent years, wireless sensor networks have been widely used in coal mine safety detection system, and the effect is remarkable, which effectively improves the efficiency of coal mine operation and reduces casualties and other accidents.

\section{Acknowledgements}

Research project of Gansu Higher Education Institute, project number: 2018C-25.

\section{References}

[1] Huang, Fangfang., Huang, Hai, Army. coal mine safety monitoring system based on wireless sensor networks. Computer Programming Skills and Maintenance, no. 24, pp. 84-86, 2017.

[2] Huang, Buyi., Chang, runner-up., Zhang, Haixia., Wang, Jun. Design of coal mine safety monitoring system based on wireless sensor network. Communications Technology, no. 09, pp. 176-178, 2008.

[3] Wang, Shuai., Shao, Mingjie., Zhang, Wanze., Tong, Dejun., Yuan, Ziyun., Zhang, Xu., Xue, Tao. Coal mine safety monitoring system based on wireless sensor networks. China New Communications, no. 21, pp. 15-17, 2007.

[4] Zhang, Xuewen. Discussion of coal mine safety monitoring system based on wireless sensor network. Value Engineering, no. 06, pp. 147, 2012. 
[5] Dai, Yunjie. coal mine safety monitoring system based on wireless sensor networks. Shanghai Normal University, 2009.

[6] Ye, Peishun., Liu, Li., Wang, Bohuai. Research and design of coal mine safety monitoring system based on wireless sensor network. Electronic Design Engineering, no. 18, pp. 67-70, 2013. 